\title{
Preischemic Treadmill Exercise Ameliorates Memory Impairment and Microvasculature Damage in Rat Model of Chronic Cerebral Hypoperfusion
}

\author{
Jae-Min Lee ${ }^{1}$, Jongmin Park ${ }^{2}$, Joo-Hee Lee ${ }^{3}$, Min Kyung Song ${ }^{4}$, Youn-Jung Kim ${ }^{1}$ \\ ${ }^{1}$ College of Nursing Science, Kyung Hee University, Seoul, Korea \\ ${ }^{2}$ College of Nursing, Research Institute of Nursing Science, Pusan National University, Yangsan, Korea \\ ${ }^{3}$ Department of Nursing, Graduate School, Kyung Hee University, Seoul, Korea \\ ${ }^{4}$ Robert Wood Johnson Medical School Institute for Neurological Therapeutics, Rutgers Biomedical and Health Sciences, Newark, NJ, USA
}

Purpose: Silent information regulator 1 (SIRT1) in the brain is essential for maintaining cellular homeostasis and plays a neuroprotective role in cerebral ischemia and neurodegenerative disorders. The effect of preischemic treadmill exercise on chronic cerebral hypoperfusion (CCH)-induced spatial learning memory impairment, microvascular injury, and blood-brain barrier (BBB) disruption in relation with SIRT1 expression was evaluated.

Methods: Prior to bilateral common carotid artery occlusion (BCCAO) surgery, the rats in the exercise groups performed low-intensity treadmill running for 30 minutes once daily during 8 weeks. BCCAO surgery was performed on male Wistar rats at 12 weeks of age. Spatial learning memory was measured using the Morris water maze test. Neuronal nuclear antigen, SIRT1, and rat endothelial cells antigen 1 were determined by immunohistochemistry and platelet-derived growth factor receptor beta was determined by immunofluorescence.

Results: Preischemic treadmill exercise ameliorated spatial learning memory impairment and enhanced SIRT1 expression in the $\mathrm{BCCAO}$ rats. Preischemic treadmill exercise ameliorated $\mathrm{BCCAO}$-induced damage to microvasculature and pericytes that make up the BBB. The effect of preischemic treadmill exercise was lost with sirtinol treatment.

Conclusions: These results can apply treadmill exercise prior to cerebral ischemia as a rational preventive and therapeutic intervention strategy to improve cognitive dysfunction in $\mathrm{CCH}$ patients.

Keywords: Chronic cerebral hypoperfusion; Bilateral common carotid artery occlusion; Preischemic treadmill exercise; Silent information regulator 1

- Fund/Grant Support: This study was supported by the Ministry of Education of the Republic of Korea and the National Research Foundation of Korea (NRF-2016S1A5B5A01022873 and NRF-2019R1I1A1A01060991).

- Research Ethics: We obtained the approval from the Kyung Hee University Institutional Animal Care and Use Committee (KHUASP [SE]-20146).

- Conflict of Interest: No potential conflict of interest relevant to this article was reported.

\footnotetext{
- HIGHLIGHTS

- Preischemic treadmill exercise improved BCCAO-induced spatial learning memory impairment.

- SIRT1 expression was upregulated by preischemic treadmill exercise following BCCAO.

- Preischemic treadmill exercise ameliorated damage to microvasculature and pericytes that make up the BBB.
}

Corresponding author: Youn-Jung Kim (D) https://orcid.org/0000-0003-4991-0825 Department of Basic Nursing Science, College of Nursing Science, Kyung Hee University, 26 Kyunghee-daero, Dongdaemun-gu, Seoul 02447, Korea Email: yj129@khu.ac.kr

Submitted: September 10, 2021 / Accepted after revision: October 29, 2021
This is an Open Access article distributed under the terms of the Creative Commons Attribution Non-Commercial License (http://creativecommons.org/licenses/by-nc/4.0/) which permits unrestricted non-commercial use, distribution, and reproduction in any medium, provided the original work is properly cited. 


\section{INTRODUCTION}

In the senile dementia, vascular dementia is the 2nd etiology after Alzheimer disease [1]. Vascular dementia is a type of neurodegenerative disease caused by a thromboembolism that reduces the amount of blood pumped to the brain. The reduction in brain blood flow induces oxidative stress and destroys the blood-brain barrier (BBB), leading to cognitive impairment [2]. In chronic cerebral hypoperfusion (CCH) studies, permanent bilateral common carotid artery occlusion (BCCAO) is a wellknown vascular dementia model. BCCAO caused $\mathrm{BBB}$ disruption and increased permeability, resulting in neurovascular dysfunction $[3,4]$.

Microvascular cells of the brain are essential components of the neurovascular unit because they structurally form the BBB and play various necessary roles in angiogenesis, cerebral blood flow regulation, and immune responses [5]. Perivascular cell dysfunction leads to the onset and exacerbation of various neurodegenerative diseases, including stroke and Alzheimer's disease. $\mathrm{BBB}$ is an essential diffusion barrier that prevents toxic substances entering the brain from the blood circulation and BBB is supported by pericytes and astrocytes [6]. BBB disruption compromises the permeability barrier formed by endothelial cells surrounded by pericytes, astrocyte end-feet, and the basal lamina. The pericytes composes the capillary wall and act as important regulators, interacting directly with the endothelial cells of the neurovascular unit. Injury to pericytes increases the permeability of the BBB, leading to release of matrix metallopeptidase-9 $[7,8]$.

Silent information regulator 1 (SIRT1) is a nicotinamide adenine dinucleotide-dependent histone deacetylase regulating cell growth and DNA damage repair, stress resistance, and apoptosis. SIRT1 exerts protective role on cerebral ischemia and neurodegenerative diseases. Activation of SIRT1 through resveratrol, calorie restriction, and exercise is involved in various biological processes by deacetylating numerous transcription factors $[9,10]$. Activated SIRT1 regulated neuronal apoptosis and energy metabolism, thereby increased the anti-oxidative stress ability of cells, providing neuroprotective effect of neuronal cells in the brain [10].

Exercise has beneficial effect on brain health and cognitive function and exercise has been considered effective in preventing and treating neurodegenerative diseases [11]. Low-intensity treadmill exercise training after $\mathrm{CCH}$ improved angiogenesis and neurogenesis, reduced neuroinflammation, and main- tained BBB and neurovascular units [12,13]. Exercise-induced overexpression of SIRT1 exerted neuroprotective effect on neuroinflammation of Parkinson's disease, Alzheimer's disease, and ischemic stroke animal models [14]. The effect of SIRT1 activation by preischemic treadmill exercise on a vascular dementia model has not yet been established. In the current study, the effect of preischemic treadmill exercise on impairment of spatial learning memory, injury of microvascular, and disruption of BBB in relation with SIRT1 activation in the BCCAO-induced rats was investigated.

\section{MATERIALS AND METHODS}

\section{Experimental Groups and Drug Administration}

For this study, 4-week-old male Wistar rats in (weight $80 \pm 10 \mathrm{~g}$ ) were purchased from Orient Bio (Seongnam, Korea). We randomly divided the rats into 4 groups: the sham group, the BC$\mathrm{CAO}$ group, the $\mathrm{BCCAO}$ with preischemic treadmill exercise group, and the BCCAO with preischemic treadmill exercise and sirtinol (SIRT1 inhibitor) treatment group. One week before BCCAO surgery (11 weeks in age), $10 \mathrm{mg} / \mathrm{kg}$ of sirtinol was treated intraperitoneally daily for 5 days in the rats of sirtinol treatment group. Approval number (KHUASP[SE]-20-146) was obtained from the Institutional Animal Care and Use Committee of Kyung Hee University.

\section{Bilateral Common Carotid Artery Occlusion}

BCCAO surgery was conducted at 12 weeks in age $(300 \pm 30 \mathrm{~g})$. Rats were deeply anesthetized with $2 \%$ isoflurane dissolved in a mixed gas of $70 \% \mathrm{~N}_{2} \mathrm{O}$ and $30 \% \mathrm{O}_{2}$. After a midline incision in the neck, right common carotid artery was ligated with 3-0 silk and occlusion of left common carotid artery was also done in the same manner 1 week after surgery of right common carotid artery occlusion. Rats in the sham group done the same surgical procedure without vascular ligation.

\section{Preischemic Treadmill Exercise Protocol}

Before BCCAO surgery, rats were engaged on a low-intensity treadmill for 30 min during 8 weeks, as previously described method [13]. The intensity of exercise was noninclined treadmill at $2 \mathrm{~m} / \mathrm{min}$ for the first 5 minutes, $3 \mathrm{~m} / \mathrm{min}$ for the next 5 minutes, and then $5 \mathrm{~m} / \mathrm{min}$ for the last 20 minutes. Rats in the sham group and BCCAO group stayed on the treadmill for 30 minutes without running for 30 minutes during 8 weeks. 


\section{Morris Water Maze Test}

Two weeks after BCCAO, hippocampus-dependent spatial learning memory was detected using the Morris water maze test. A circular swimming pool (diameter, $200 \mathrm{~cm}$ ) filled with water $\left(22^{\circ} \mathrm{C} \pm 2^{\circ} \mathrm{C}\right)$ to a depth of $30 \mathrm{~cm}$ was divided by an imaginary line into quadrants of equal size. The platform (diameter, $15 \mathrm{~cm}$ ) was hidden at a depth of $2 \mathrm{~cm}$ from the water surface in the center of the southeast quadrant. The walls of the test environment were given some visual cues. Training to find hidden platforms was conducted for all groups 3 times a day for 4 consecutive days. Morris water maze test was done to assess spatial learning memory the day after the last training. The time to reach the hidden platform and the time spent in the quadrant with the hidden platform were recorded.

\section{Immunohistochemistry}

Upon completion of the behavioral experiment, the mice were anesthetized. Rats were infused with $0.05 \mathrm{M}$ phosphate-buffered saline (PBS), and then treated with $4 \%$ paraformaldehyde (PFA) for fixation in $0.01 \mathrm{M}$ phosphate buffer using a cardiac catheter. Whole brains were removed and immersed in 4\% PFA for fixation for 12 hours and then immersed in 30\% sucrose solution for 3 days. Using a frozen microtome (CM3050S, Leica, Nussloch, Germany), the brains were frozen sectioned in the coronal direction to a thickness of $40 \mu \mathrm{m}$. Immunohistochemistry was conducted for neuronal nuclear antigen (NeuN), rat endothelial cells antigen 1 (RECA-1), and SIRT1 in the hippocampus and motor cortex.

Free-floating sections were incubated with $3 \% \mathrm{H}_{2} \mathrm{O}_{2}$ for 30 minutes, and the sections were incubated with blocking solution ( $1 \%$ bovine serum albumin) with $10 \%$ normal goat serum for 1 hour at room temperature. The sections were treated with NeuN antibody (1:1,000; Abcam, Cambridge, UK), RECA-1 antibody (1:1,000; Abcam), and SIRT1 antibody (1:500; Abcam) overnight at $4^{\circ} \mathrm{C}$. The sections were treated with the biotinylated mouse secondary antibodies (1:200; Vector Laboratories, Burlingame, CA, USA) with $0.3 \%$ Triton X-100 (Vector Laboratories) in PBS for 1 hour and subsequently treated with antibody avidin-biotin-peroxidase complex solution (Vector Laboratories) for 1 hour at room temperature. Finally, the sections were stained by diaminobenzidine tetrahydrochloride (Vector Laboratories). After the sections were attached to gelatin-coated slides, the slides were air-dried overnight at room temperature, and coverslips were mounted using a Permount (Vector Laboratories). The number of cells in all samples was calculated by optical microscope (Nikon Corporation, Tokyo, Japan).

\section{Immunofluorescence}

For the immunofluorescence staining, the sections were treated with one of the following primary antibodies: mouse monoclonal antibody to RECA- 1 antibody (1:1,000; Abcam) and rabbit monoclonal antibody to platelet-derived growth factor receptor beta (PDGFR $\beta$ ) (1:500; Abcam) overnight at $4 \mathrm{oC}$ after blocking. Alexa Fluor 594-conjugated anti-mouse IgG (1:1,000; Molecular Probes, Eugene, OR, USA) and Alexa Fluor 488-conjugated anti-rabbit IgG (1:1,000; Molecular Probes) were treated to the sections for 1 hour at room temperature. Alexa Fluor 594 (red) and Alexa Fluor 488 (green) fluorescence photographs were observed with a confocal microscope (Zeiss LSM 700; Zeiss, Oberkochen, Germany). Image-Pro Plus software (Media Cybernetics, Silver Spring, MD, USA) was used for data analysis.

\section{Statistical Analysis}

Data were analyzed using IBM SPSS Statistics ver. 25.0 (IBM Co., Armonk, NY, USA), and results were presented as the mean \pm standard error of the mean. The latency of the Morris water maze test for 4 consecutive days were analyzed by 2 -way repeated-measures analysis of variance (ANOVA). One-way ANOVA was used for other data analysis, and significance was measured by Tukey post hoc test. $\mathrm{P}<0.05$ showed statistical significance.

\section{RESULTS}

\section{Spatial Learning Memory}

BCCAO caused vascular dementia-like deficits. The current study evaluated the effect of preischemic treadmill exercise on BCCAO-induced spatial learning memory impairment using the Morris water maze test (Fig. 1). The latency time to find the hidden platform was increased in the BCCAO-induced rats compared to the control rats $(\mathrm{P}<0.05)$. In contrast, the latency time was decreased by preischemic treadmill exercise in the BCCAO-induced rats $(\mathrm{P}<0.05)$. The time spent $(\%)$ in the quadrant with the hidden platform was shortened in the $\mathrm{BC}$ CAO-induced rats compared to the control rats $(\mathrm{P}<0.05)$. In contrast, the time spent was lengthened by preischemic treadmill exercise in the BCCAO-induced rats $(\mathrm{P}<0.05)$. In addition, sirtinol, a SIRT1inhibitor, treatment reduced the effect of 

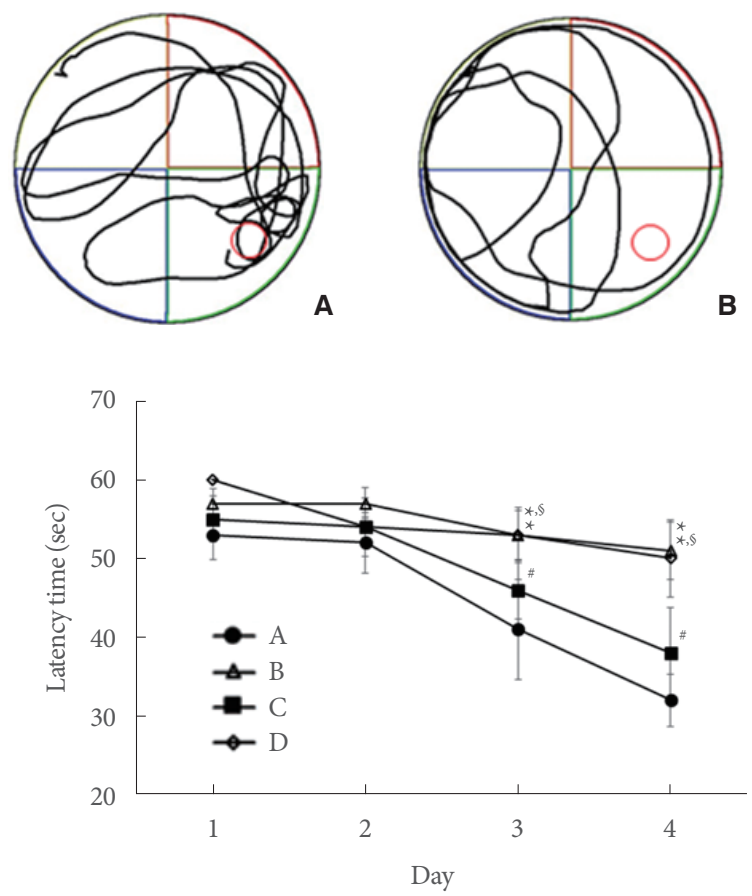
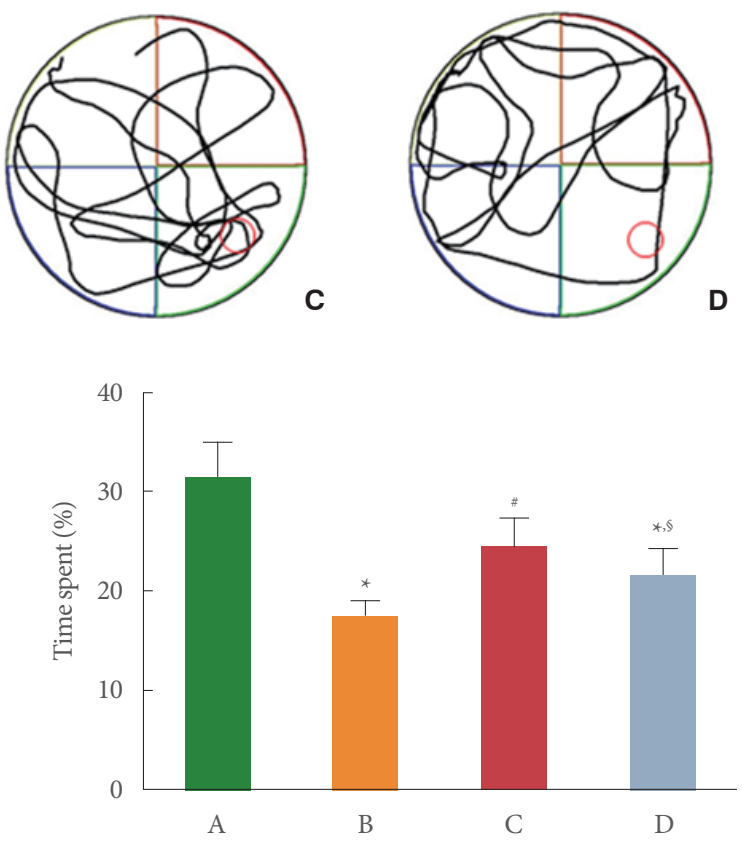

Fig. 1. Effect of preischemic treadmill exercise on bilateral common carotid artery occlusion (BCCAO)-induced spatial learning memory impairment. Upper panel: representative tracking of swimming path. Lower left panel: latency time to find hidden platform. Lower right panel: \% time spent in the target platform quadrant. A, sham group; B, BCCAO group; C, BCCAO with preischemic treadmill exercise group; $\mathrm{D}, \mathrm{BCCAO}$ with preischemic treadmill exercise and sirtinol treatment group. ${ }^{\star} \mathrm{P}<0.05$ compared to sham group. ${ }^{*} \mathrm{P}<0.05$ compared to $\mathrm{BCCAO}$ group. ${ }^{5} \mathrm{P}<0.05$ compared to $\mathrm{BCCAO}$ with preischemic treadmill exercise group.

preischemic treadmill exercise.

\section{Neuronal Marker}

NeuN is well known as a neuronal marker, and NeuN immunostaining in the hippocampal cornu Ammonis 1 (CA1) and cornu Ammonis 3 (CA3) region is shown in Fig. 2. After BCCAO surgery, the number of NeuN-positive cells in the hippocampal CA1 and CA3 region was decreased in the BCCAO-induced rats compared to the control rats, but there was no statistically significant change.

\section{SIRT1 Expression}

SIRT1 expression in the hippocampal CA1 and CA3 region is shown in Fig. 3. After BCCAO surgery, the number of SIRT1positive cells in the hippocampal CA1 and CA3 region was decreased in the BCCAO-induced rats compared to the control rats $(\mathrm{P}<0.05)$. However, the number of SIRT1-positive cells was increased by preischemic treadmill exercise in the BCCAO-induced rats $(\mathrm{P}<0.05)$. In addition, sirtinol treatment abrogated the effect of preischemic treadmill exercise.

\section{Microvasculature Damage}

RECA-1 immunostaining in the motor cortex is shown in Fig. 4. The number of fragments of microvasculature of RECA- 1 less than $30 \mu \mathrm{m}$ in the motor cortex was measured. After BCCAO surgery, the number of fragments less than $30 \mu \mathrm{m}$ was increased $(\mathrm{P}<0.05)$ and the length of microvasculature was shortened $(\mathrm{P}<0.05)$. Meanwhile, preischemic treadmill exercise reduced the number of fragments less than $30 \mu \mathrm{m}(\mathrm{P}<0.05)$ and increased microvasculature length $(\mathrm{P}<0.05)$. These results showed that preischemic treadmill exercise ameliorated BCCAO-induced microvasculature damage. In addition, sirtinol treatment made the effect of exercise disappear.

\section{BBB Disruption}

Immunofluorescence staining of PDGFR $\beta$ expression in the motor cortex is shown Fig. 5. After BCCAO surgery, PDGFR $\beta$ expression in the motor cortex was decreased in the $\mathrm{BCCAO}$-induced rats compared to the control rats $(\mathrm{P}<0.05)$. But, PDGFR $\beta$ expression was increased by preischemic treadmill exercise in the BCCAO-induced rats $(\mathrm{P}<0.05)$. In addition, sirtinol treatment made the effect of exercise disappear. 
A

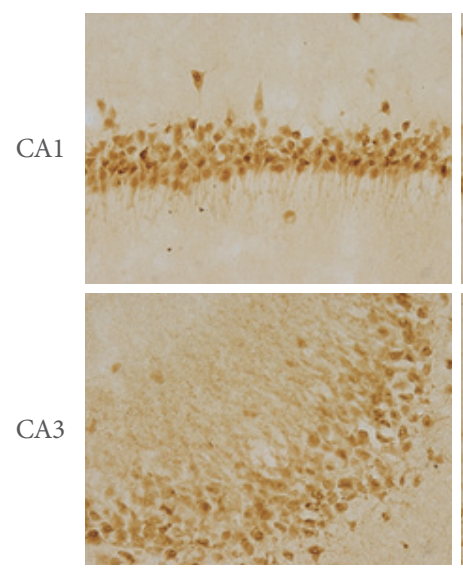

$\mathrm{B}$
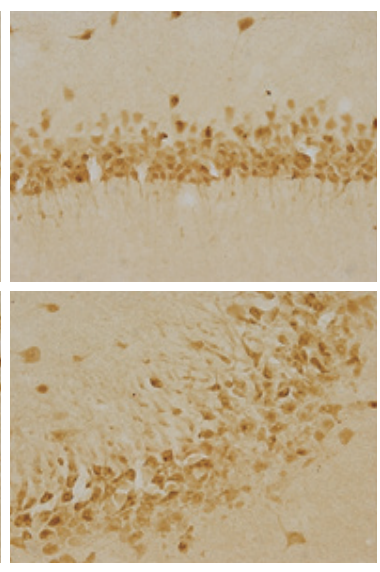

C
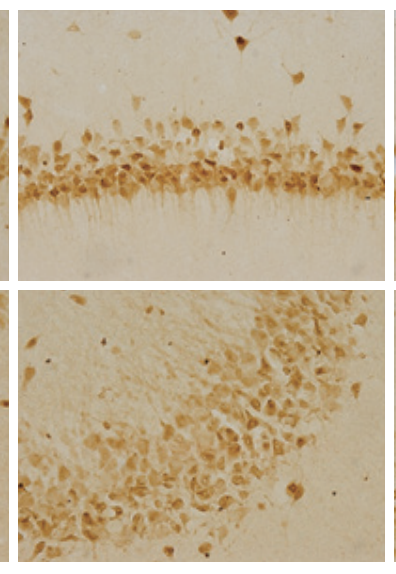

$\mathrm{D}$
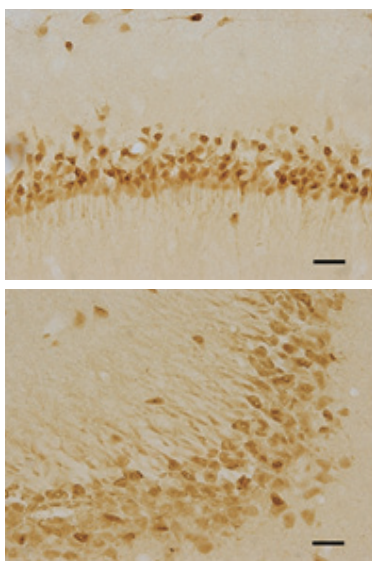
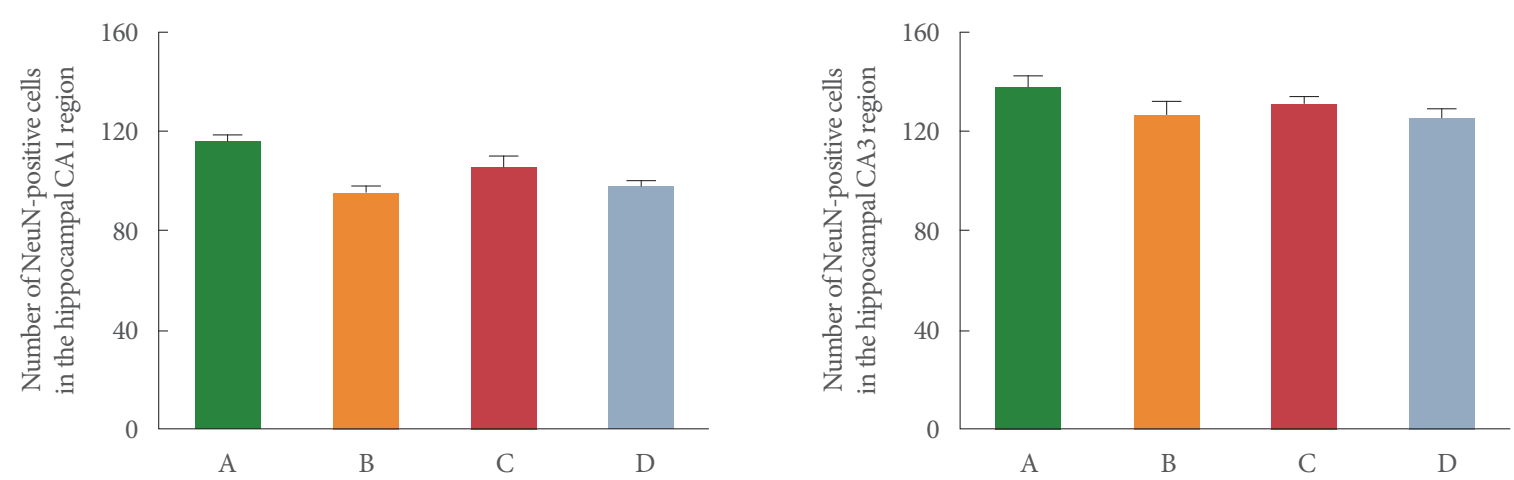

Fig. 2. Effect of preischemic treadmill exercise on bilateral common carotid artery occlusion (BCCAO)-induced neuronal cell death in the hippocampal cornu Ammonis 1 (CA1) and cornu Ammonis (CA3) region. Upper panel: representative photomicrographs of immunostaining of neuronal nuclear antigen (NeuN)-positive cells in the hippocampal CA1 and CA3 region. The scale bar indicates $50 \mu \mathrm{m}$. Lower left panel: number of NeuN-positive cells in hippocampal CA1 region. Lower right panel: number of NeuN-positive cells in hippocampal CA3 region. A, sham group; B, BCCAO group; C, BCCAO with preischemic treadmill exercise group; D, BC$\mathrm{CAO}$ with preischemic treadmill exercise and sirtinol treatment group.

\section{DISCUSSION}

BCCAO-induced $\mathrm{CCH}$ caused cerebral hypoxia, resulting in cognitive deficits in the rats [15]. Treadmill exercise improved cognitive function in several neurodegenerative diseases [16, 17]. Exercise exerted neuroprotective effect and enhanced synaptic plasticity and neurogenesis by reducing neuroinflammation and oxidative stress in stroke $[11,18]$. The present study also confirmed that $\mathrm{CCH}$-induced by BCCAO in the Morris water maze test resulted in spatial learning impairment. Preischemic exercise improved memory deficits caused by $\mathrm{CCH}$. However, performing preischemic exercise with sirtinol treatment, the effect of exercise did not appear.

We confirmed the BCCAO-induced neuronal damage through staining of NeuN-positive cells in the hippocampus and motor cortex. There was no statistical difference in any group. The number of NeuN-positive cells in the CA1 region of the hippocampus was significantly reduced by $10 \%-15 \%$ during 4 weeks after BCCAO [19]. However, several studies reported that the number of NeuN-positive cells in the motor cortex and hippocampus was decreased by about $10 \%$ compared with the control group after BCCAO, but there was no statistically significant difference $[20,21]$. From these results, it could be seen that the neuronal damage by BCCAO was not so great.

SIRT1 is essential for maintaining cellular homeostasis by regulating inflammatory and oxidative stressor mechanisms and plays a neuroprotective role in cerebral ischemia and neurodegenerative disorders. Although SIRT1 can exert a wide range of neuroprotective effects, it has been reported that the degree of protection by SIRT1 overexpression is at most partial [22]. 
A
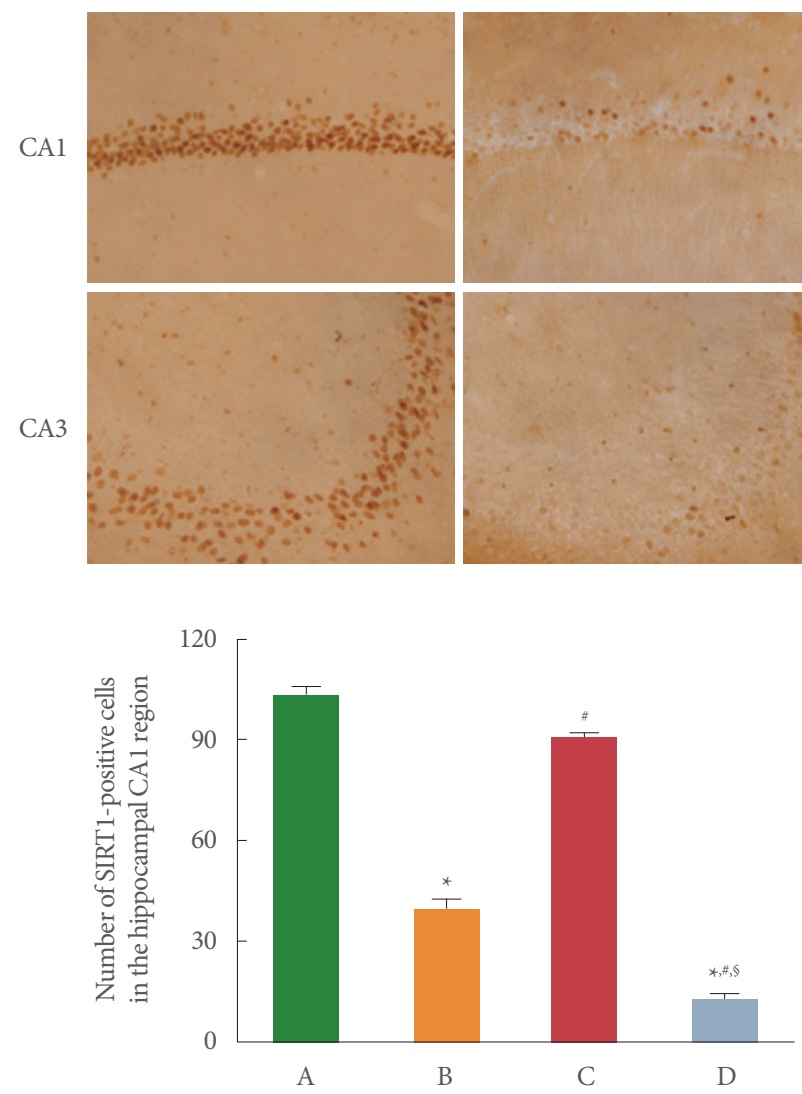

B
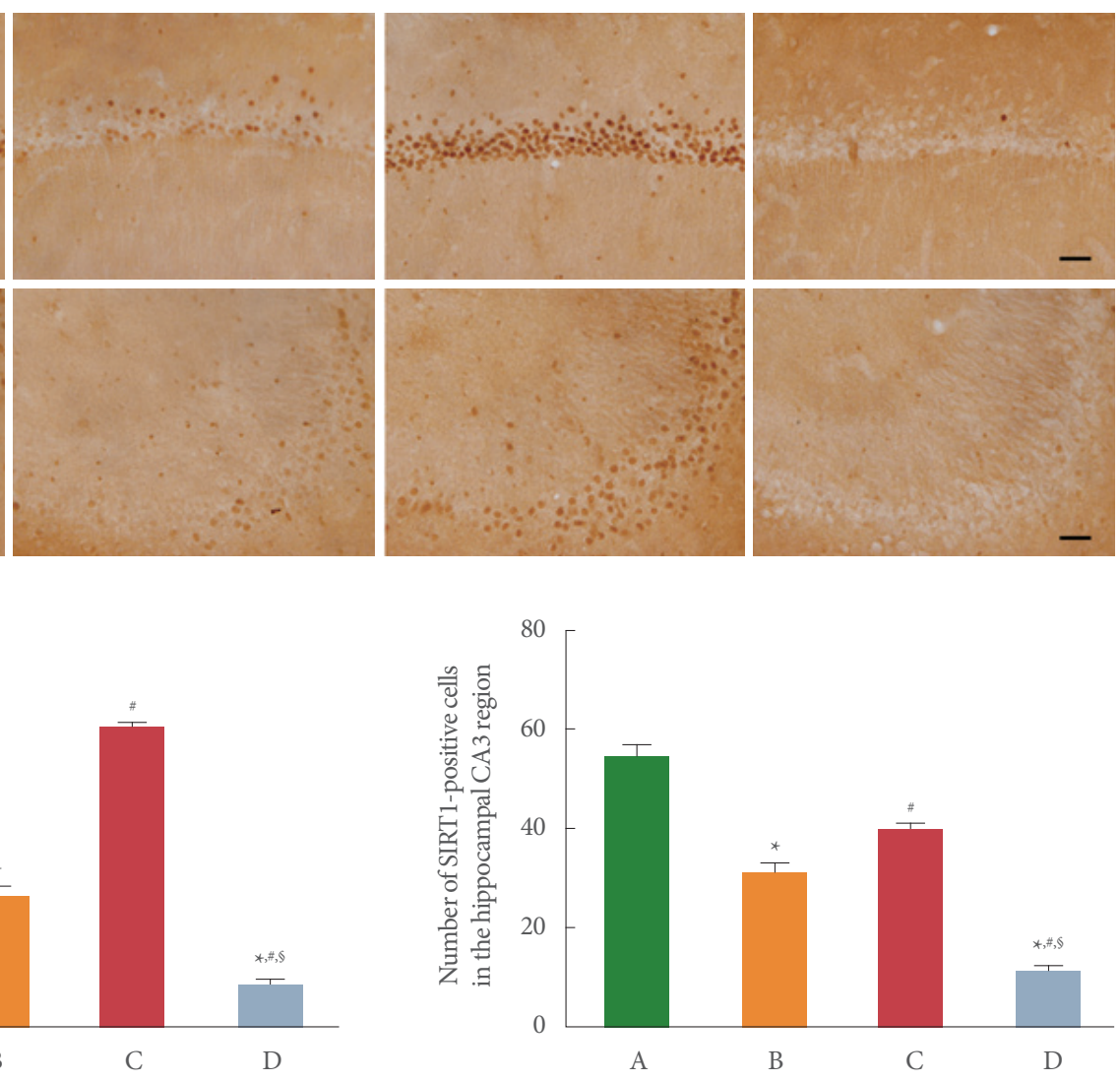

Fig. 3. Effect of preischemic treadmill exercise on bilateral common carotid artery occlusion (BCCAO)-induced silent information regulator 1 (SIRT1) expression in the hippocampal cornu Ammonis 1 (CA1) and cornu Ammonis (CA3) region. Upper panel: representative photomicrographs of immunostaining of SIRT1-positive cells in the hippocampal CA1 and CA3 region. The scale bar indicates $50 \mu \mathrm{m}$. Lower left panel: number of SIRT1-positive cells in the hippocampal CA1 region. Lower right panel: number of SIRT1positive cells in the hippocampal CA3 region. A, sham group; B, BCCAO group; C, BCCAO with preischemic treadmill exercise group; $\mathrm{D}, \mathrm{BCCAO}$ with preischemic treadmill exercise and sirtinol treatment group. ${ }^{*} \mathrm{P}<0.05$ compared to sham group. ${ }^{*} \mathrm{P}<0.05$ compared to $\mathrm{BCCAO}$ group. ${ }^{\mathrm{P}} \mathrm{P}<0.05$ compared to $\mathrm{BCCAO}$ with preischemic treadmill exercise group.

High level of SIRT1 expression in the brain indicates normal functioning of synaptic plasticity and memory, suggesting that SIRT1 is essential for learning ability, memory process, and maintaining of synaptic plasticity in mice [23]. Treadmill exercise improved memory impairment by increasing SIRT1 expression [24]. Long-term moderate treadmill exercise ameliorated cognitive dysfunction by activating SIRT1 to alleviate inflammatory response in the hippocampus [25]. SIRT1 inhibitor treatment displayed cognitive impairment and synaptic plasticity deficiency, whereas overexpression of SIRT1 through exercise had neuroprotective effects in the hippocampus of Alzheimer's disease mice [26]. In the current study, SIRT1 expression was reduced in the hippocampus by $\mathrm{CCH}$ and upregulated by preischemic treadmill exercise. It was confirmed that the ex- pression of SIRT1 was disappeared by sirtinol treatment. The current results demonstrated that preischemic treadmill exercise alleviated $\mathrm{CCH}$-induced memory impairment through SIRT1 activation.

RECA-1 is used as a vascular endothelial cell-specific biomarker. Damage to microvasculature in the brain after $\mathrm{CCH}$ leads to cognitive impairment. In the previous study, treadmill exercise also alleviated $\mathrm{CCH}$-induced microvascular damage and cognitive impairment [13]. In the present study, the number of fragments smaller than $30 \mu \mathrm{m}$ of RECA- 1 was increased and the length of RECA-1 was shortened by $\mathrm{CCH}$, demonstrating that $\mathrm{CCH}$-induced damage to the microvasculature in the cortex. By the preischemic treadmill exercise, the number of fragments smaller than $30 \mu \mathrm{m}$ of RECA- 1 was reduced and the 
A
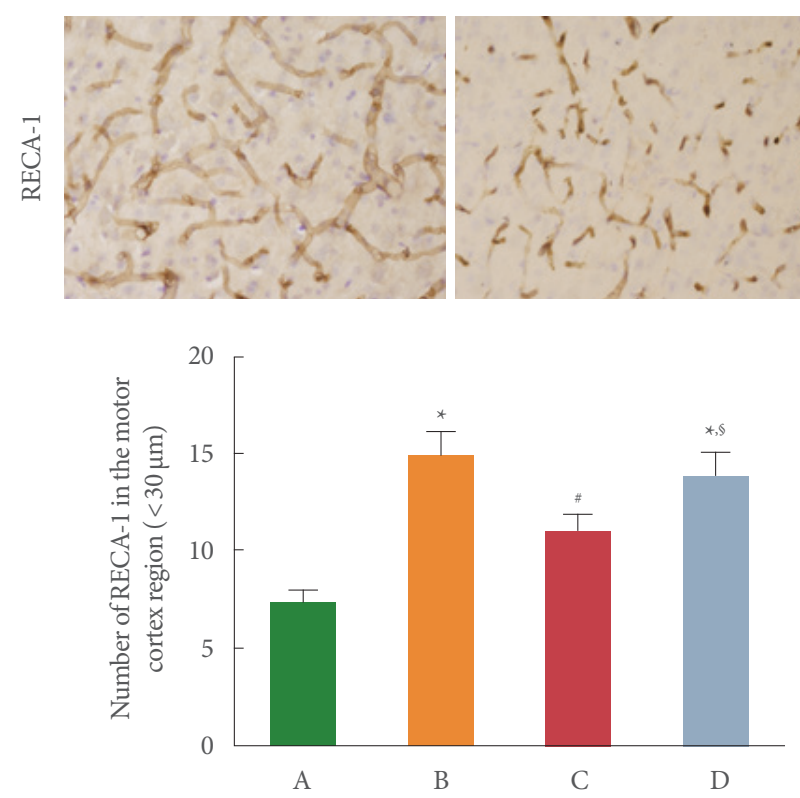

C
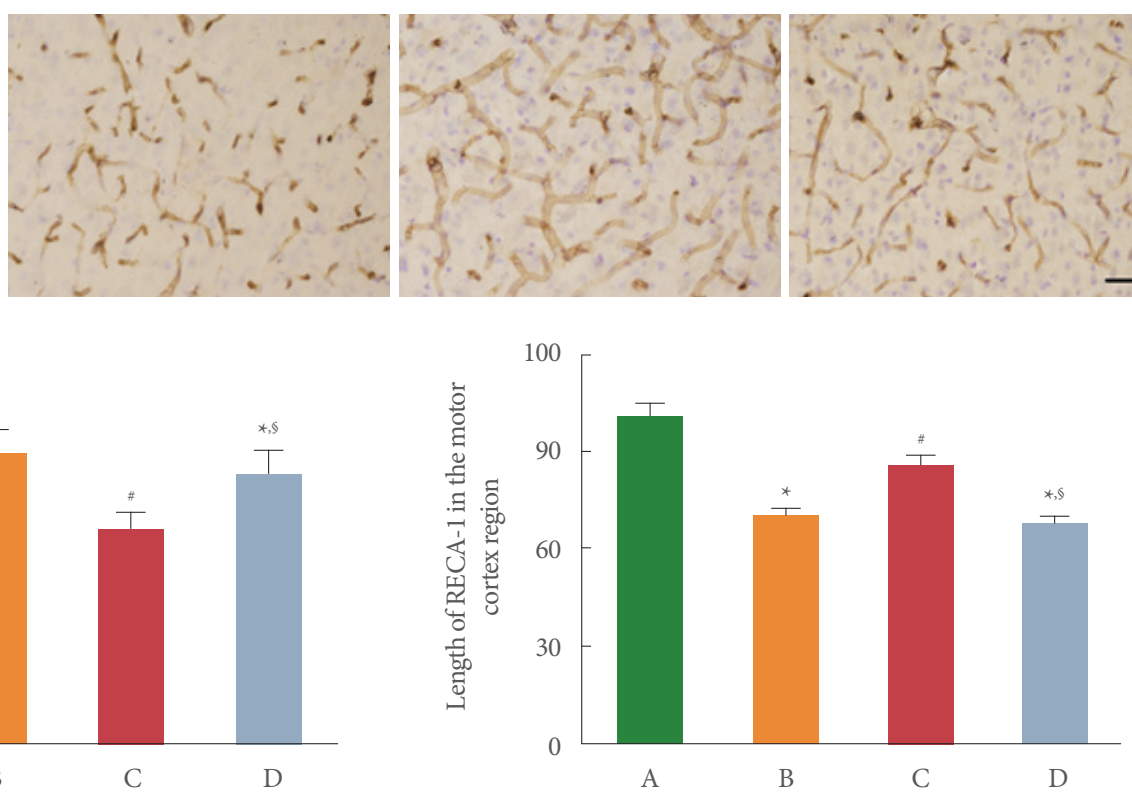

Fig. 4. Effect of preischemic treadmill exercise on bilateral common carotid artery occlusion (BCCAO)-induced microvasculature damage in the motor cortex. Upper panel: representative photomicrographs of immunostaining of rat endothelial cells antigen 1 (RECA-1) in the motor cortex. The scale bar indicates $50 \mu \mathrm{m}$. Lower left panel: number of RECA-1 in the motor cortex. Lower right panel: length of RECA-1 in the motor cortex. A, sham group; B, BCCAO group; C, BCCAO with preischemic treadmill exercise group; $\mathrm{D}, \mathrm{BCCAO}$ with preischemic treadmill exercise and sirtinol treatment group. ${ }^{\star} \mathrm{P}<0.05$ compared to sham group. ${ }^{*} \mathrm{P}<0.05$ compared to $\mathrm{BCCAO}$ group. ${ }^{5} \mathrm{P}<0.05$ compared to $\mathrm{BCCAO}$ with preischemic treadmill exercise group.
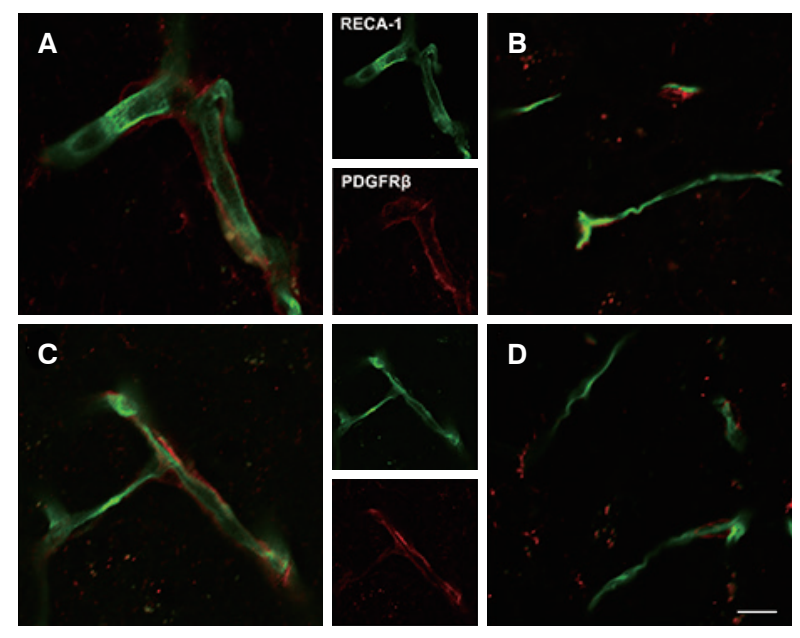
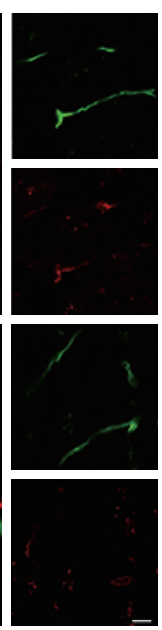

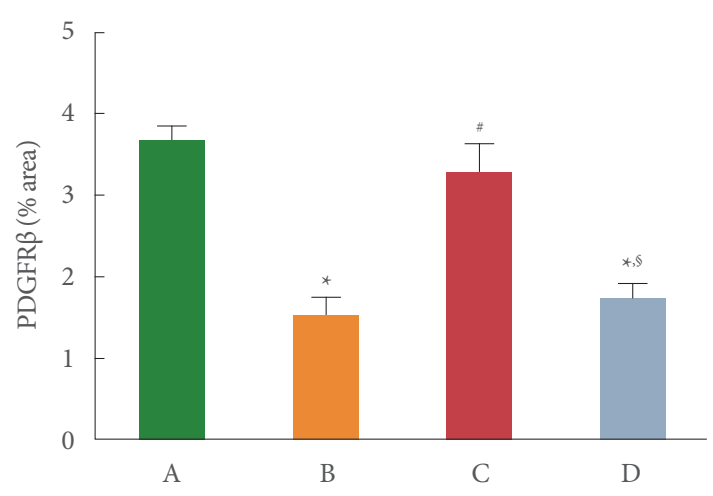

Fig. 5. Effect of preischemic treadmill exercise on bilateral common carotid artery occlusion (BCCAO)-induced pericytes damage in the motor cortex. Left panel: representative photomicrographs of immunofluorescence staining of rat endothelial cells antigen 1 (RECA-1) (green) and platelet-derived growth factor receptor beta (PDGFR $\beta$ ) (red) in the motor cortex. The scale bars in the large square and small square figures are all $20 \mu \mathrm{m}$. Right panel: \% area of PDGFR $\beta$ in the motor cortex. A, sham group; B, BCCAO group; $\mathrm{C}, \mathrm{BCCAO}$ with preischemic treadmill exercise group; $\mathrm{D}, \mathrm{BCCAO}$ with preischemic treadmill exercise and sirtinol treatment group. ${ }^{\star} \mathrm{P}<0.05$ compared to sham group. ${ }^{*} \mathrm{P}<0.05$ compared to $\mathrm{BCCAO}$ group. ${ }^{\varsigma} \mathrm{P}<0.05$ compared to $\mathrm{BCCAO}$ with preischemic treadmill exercise group. 
length of RECA-1 was increased again, demonstrating that microvasculature damage was improved by preischemic treadmill exercise. However, sirtinol treatment did not alleviate damage to the microvasculature. These results suggest that preischemic treadmill exercise can ameliorate $\mathrm{CCH}$-induced microvascular damage.

PDGFR $\beta$ plays an essential role in BBB functional recovery and integrity after BCCAO. BBB dysfunction is known to be caused by $\mathrm{CCH}$-induced neuroinflammation and increased oxidative stress [27]. Therefore, the BBB could be a potential therapeutic target for cognitive impairment due to $\mathrm{CCH}$-induced neuroinflammation, nerve damage, and increased oxidative stress $[28,29]$. In the cerebral vessels, BBB disruption precedes the pathogenesis of vascular dementia [30]. Pericytes are important regulators of maturation, homeostasis, and maintenance of cerebrovascular function. Loss of PDGFR $\beta$ (pericyte marker) appears in the neurological disorders that increase BBB permeability. PDGFR $\beta$-deficient mice showed markedly reduced microvessel length in the cortex, hippocampus, and other brain regions [31]. In the current study, PDGFR $\beta$ expression was decreased after $\mathrm{CCH}$, but PDGFR $\beta$ expression was increased by preischemic treadmill exercise. However, the PDGFR $\beta$-increasing effect of preischemic treadmill exercise was not shown by sirtinol treatment.

In the current results, preischemic treadmill exercise improved spatial learning memory and enhanced SIRT1 expression in the BACCAO rats. Preischemic treadmill exercise ameliorated BC$\mathrm{CAO}$-induced damage to microvasculature and pericytes that make up the BBB. Treadmill exercise before cerebral ischemia can be used as a rational preventive and therapeutic intervention strategy to ameliorate cognitive dysfunction in patients with this $\mathrm{CCH}$.

\section{AUTHOR CONTRIBUTION STATEMENT}

- Conceptualization: JML

- Data curation: JP, JHL

- Formal analysis: JP, JHL

- Funding acquisition: $Y J K$

- Methodology: JML

- Project administration: $Y J K$

- Visualization: $M K S$

- Writing-original draft: $J M L$

-Writing-review \& editing: $Y J K$

\section{ORCID}

$\begin{array}{ll}\text { Jae-Min Lee } & 0000-0002-7546-9765 \\ \text { Jongmin Park } & 0000-0001-6176-5670 \\ \text { Joo-Hee Lee } & 0000-0001-7446-2214 \\ \text { Min Kyung Song } & 0000-0002-1779-5992 \\ \text { Youn-Jung Kim } & 0000-0003-4991-0825\end{array}$

\section{REFERENCES}

1. Román GC. Vascular dementia may be the most common form of dementia in the elderly. J Neurol Sci 2002;203-204:7-10.

2. Venkat P, Chopp M, Chen J. Models and mechanisms of vascular dementia. Exp Neurol 2015;97-108.

3. Cai ZY, Yan Y, Sun SQ, Zhang J, Huang LG, Yan N, et al. Minocycline attenuates cognitive impairment and restrains oxidative stress in the hippocampus of rats with chronic cerebral hypoperfusion. Neurosci Bull 2008;24:305-13.

4. Lee JM, Lee JH, Song MK, Kim YJ. NXP031 improves cognitive impairment in a chronic cerebral hypoperfusion-induced vascular dementia rat model through nrf2 signaling. Int J Mol Sci 2021;22: 6285.

5. Sá-Pereira I, Brites D, Brito MA. Neurovascular unit: a focus on pericytes. Mol Neurobiol 2012;45:327-47.

6. Dingezweni S. The blood-brain barrier. S Afr J Anaesth Analg 2020;26:S32-4.

7. Brown LS, Foster CG, Courtney JM, King NE, Howells DW, Sutherland BA. Pericytes and neurovascular function in the healthy and diseased brain. Front Cell Neurosci 2019;13:282.

8. Zhang W, Zhu L, An C, Wang R, Yang L, Yu W, et al. The blood brain barrier in cerebral ischemic injury - Disruption and repair. Brain Hemorrhages 2020;1:34-53.

9. Ferrara N, Rinaldi B, Corbi G, Conti V, Stiuso P, Boccuti S, et al. Exercise training promotes SIRT1 activity in aged rats. Rejuvenation Res 2008;11:139-50.

10. Xu J, Jackson CW, Khoury N, Escobar I, Perez-Pinzon MA. Brain SIRT1 mediates metabolic homeostasis and neuroprotection. Front Endocrinol (Lausanne) 2018;9:702.

11. Hong M, Kim M, Kim TW, Park SS, Kim MK, Park YH, et al. Treadmill exercise improves motor function and short-term memory by enhancing synaptic plasticity and neurogenesis in photothrombotic stroke mice. Int Neurourol J 2020;24(Suppl 1):S28-38.

12. Małkiewicz MA, Szarmach A, Sabisz A, Cubała WJ, Szurowska E, Winklewski PJ. Blood-brain barrier permeability and physical exercise. J Neuroinflammation 2019;16:15. 
13. Lee JM, Baek SS, Kim TW, Park HS, Park SS, Park JM, et al. Preischemic treadmill exercise improves short-term memory by inhibiting hypoperfusion-induced disruption of blood-brain barrier after bilateral common carotid arteries occlusion. J Exerc Rehabil 2019;15:370-6.

14. Seo DY, Heo JW, Ko JR, Kwak HB. Exercise and neuroinflammation in health and disease. Int Neurourol J 2019;23(Suppl 2):S82-92.

15. Jing Z, Shi C, Zhu L, Xiang Y, Chen P, Xiong Z, et al. Chronic cerebral hypoperfusion induces vascular plasticity and hemodynamics but also neuronal degeneration and cognitive impairment. J Cereb Blood Flow Metab 2015;35:1249-59.

16. Ko YJ, Kim BK, Ji ES. Treadmill exercise in obese maternal rats during pregnancy improves spatial memory through activation of phosphatidylinositol 3-kinase pathway in the hippocampus of rat pups. J Exerc Rehabil 2020;16:483-8.

17. Park HS, Kim TW, Park SS, Lee SJ. Swimming exercise ameliorates mood disorder and memory impairment by enhancing neurogenesis, serotonin expression, and inhibiting apoptosis in social isolation rats during adolescence. J Exerc Rehabil 2020;16:132-40.

18. Lee SS, Kim CJ, Shin MS, Lim BV. Treadmill exercise ameliorates memory impairment through ERK-Akt-CREB-BDNF signaling pathway in cerebral ischemia gerbils. J Exerc Rehabil 2020;16:4957.

19. Xiong Z, Lu W, Zhu L, Zeng L, Shi C, Jing Z, et al. Dl-3-n-butylphthalide treatment enhances hemodynamics and ameliorates memory deficits in rats with chronic cerebral hypoperfusion. Front Aging Neurosci 2017;9:238.

20. Zhu Y, Zhang Q, Zhang W, Li N, Dai Y, Tu J, et al. Protective effect of $17 \beta$-estradiol upon hippocampal spine density and cognitive function in an animal model of vascular dementia. Sci Rep 2017;7:42660.

21. Song MK, Kim YJ, Lee JM, Kim YJ. Neurovascular integrative effects of long-term environmental enrichment on chronic cerebral hypoperfusion rat model. Brain Res Bull 2020;163:160-9.
22. Imai S, Guarente L. NAD+ and sirtuins in aging and disease. Trends Cell Biol 2014;24:464-71.

23. Michán S, Li Y, Chou MMH, Parrella E, Ge H, Long JM, et al. SIRT1 is essential for normal cognitive function and synaptic plasticity. J Neurosci 2010;30:9695-707.

24. Kim TW, Park SS, Shin MS, Park HS, Baek SS. Treadmill exercise ameliorates social isolation-induced memory impairment by enhancing silent information regulator-1 expression in rats. J Exerc Rehabil 2020;16:227-33.

25. Lang X, Zhao N, He Q, Li X, Li X, Sun C, et al. Treadmill exercise mitigates neuroinflammation and increases $\mathrm{BDNF}$ via activation of SIRT1 signaling in a mouse model of T2DM. Brain Res Bull 2020; 165:30-9.

26. Corpas R, Revilla S, Ursulet S, Castro-Freire M, Kaliman P, Petegnief $\mathrm{V}$, et al. SIRT1 overexpression in mouse hippocampus induces cognitive enhancement through proteostatic and neurotrophic mechanisms. Mol Neurobiol 2017;54:5604-19.

27. Sun Z, Gao C, Gao D, Sun R, Li W, Wang F, et al. Reduction in pericyte coverage leads to blood-brain barrier dysfunction via endothelial transcytosis following chronic cerebral hypoperfusion. Fluids Barriers CNS 2021;18:21.

28. Liu H, Zhang J. Cerebral hypoperfusion and cognitive impairment: the pathogenic role of vascular oxidative stress. Int J Neurosci 2012; 122:494-9.

29. Xu L, Qu C, Qu C, Shen J, Song H, Li Y, et al. Improvement of autophagy dysfunction as a potential mechanism for environmental enrichment to protect blood-brain barrier in rats with vascular cognitive impairment. Neurosci Lett 2020;739:135437.

30. Yang Y, Rosenberg GA. Blood-brain barrier breakdown in acute and chronic cerebrovascular disease. Stroke 2011;42:3323-8.

31. Bell RD, Winkler EA, Sagare AP, Singh I, LaRue B, Deane R, et al. Pericytes control key neurovascular functions and neuronal phenotype in the adult brain and during brain aging. Neuron 2010;68: 409-27. 